\title{
Eficiencia de trampas cebadas con atrayentes cairomonales y puntos cebo en la gestión de Tomicus destruens (Wollaston 1865)
}

\author{
Mas H. ${ }^{*}$, Pastor C. ${ }^{1}$, Santacruz A. ${ }^{1}$, Pérez-Laorga E. ${ }^{2}$, Marco L. ${ }^{1}$, Saiz C. ${ }^{2}$, \\ Etxebeste I. ${ }^{3}$, Gallego D.,
}

\begin{abstract}
${ }^{1}$ Laboratori de Sanitat Forestal. CIEF. VAERSA- Conselleria diInfraestructures, Territori i Medi Ambient (Generalitat Valenciana).Avda Comarques del País Valencià 114, 46930, Quart de Poblet, València.

${ }^{2}$ Conselleria diInfraestructures, Territori i Medi Ambient . Generalitat Valenciana. Ciutat administrativa 9 d'octubre. C/ Castán Tobeñas, 77. 46018 València
\end{abstract}

${ }^{3}$ Swedish University of Agricultural Sciences. Department of Ecology. Ulls väg 16, 750-07, Upssala, Suecia.

${ }^{4}$ Departamento de Zoología y Antropología Física, Universidad de Murcia, Campus Universitario de Espinardo. 30100 Murcia.

${ }^{5}$ Sanidad Agrícola ECONEX, S.L., C/ Mayor, No $15 B$ - Edificio ECONEX

Apartado de Correos $N^{\circ}$ 167, 30149 Siscar-Santomera, Murcia.

*Autor para correspondencia: lab_plagas.ctv@gva.es

\section{Resumen}

El levante de la península Ibérica ha registrado una sequía extrema desde la primavera de 2013, dando lugar a un profundo deterioro del estado fitosanitario de los pinares valencianos. Esta situación ha supuesto un aumento de la disponibilidad de material hospedante para insectos perforadores, entre ellos Tomicus destruens. El control de estos insectos se basa en dos herramientas de gestión: los puntos cebo y las trampas cebadas con atrayentes feromonales y cairomonales. Tradicionalmente los puntos cebo han sido utilizados como herramienta en la gestión de los focos de T. destruens, pero su elaboración es costosa y su manejo complicado. En los últimos años se han comercializado atrayentes cairomonales para ser utilizados en trampas como alternativa eficaz a las pilas cebo. Durante el periodo 2014-2015 se comparó la eficacia en la extracción de T. destruens entre puntos cebo y trampas (Crosstrap ${ }^{\circledR}$ y multiembudos) cebadas con atrayentes cairomonales (etanol y $\alpha$ pineno) en tres zonas de la provincia de Valencia, con el objetivo de mejorar la gestión. Las trampas resultaron más eficaces en la atracción y captura de T. destruens que los puntos cebo.

Palabras clave: $\alpha$-pineno, Crosstrap ${ }^{\circledR}$, etanol, Scolytidae, Trampa multiembudos. 


\section{Introducción}

El ataque a árboles vivos por escolítidos depende de la localización precisa de un árbol hospedador adecuado, entre los que estos insectos seleccionan frecuentemente árboles en diferentes estadios de decaimiento. La atracción hacia estos árboles decaídos está mediada en muchas especies por mono y sesquiterpenos (Fäldt, 2000), producidos por el árbol en su respuesta a la debilidad. La alta volatilidad de estos productos ha permitido por evolución que los escolítidos puedan usarlos como compuestos semioquímicos indicadores a larga distancia de decaimiento en sus hospedadores. Filella et al. (2009) estudiaron el efecto del déficit hídrico en las emisiones de compuestos orgánicos volátiles en acículas de Pinus halepensis y observaron incrementos de etanol, ácido fórmico y acetaldehído. En efecto, etanol ha sido resaltado como un compuesto semioquímico de especial relevancia en la respuesta atractiva de Tomicus destruens (Wollaston, 1865), de acuerdo con Gallego et al. (2008).

T. destruens es una especie de distribución mediterránea y macaronésica, de acuerdo con Wood and Bright (1992). Esta especie está considerada como uno de los escolítidos más relevante en los pinares mediterráneos (Guerrero et al., 1997; Sabbatini Peverieri et al., 2004; Faccoli et al., 2005; Chakali, 2005), atacando a las especies $P$. halepensis, $P$. pinaster, $P$. pinea, $P$. brutia, $P$. canariensis, $P$. radiata y muy ocasionalmente $P$. nigra (Gallego et al., 2004; Vasconcelos et al., 2006). T. destruens está ampliamente distribuido en la península Ibérica, ausente solamente en las montañas que rodean la Meseta Norte, Pirineos y las mayores altitudes de las cordilleras Béticas (Gallego et al., 2004). El vuelo reproductivo de esta especie en la península Ibérica presenta forma bimodal, con un pico en otoño y otro en el fin del invierno-primavera temprana (Gallego et al., 2004; Vasconcelos et al., 2005; Gallego, 2006; Gallego et al., 2008; Sabbatini Peverieri et al. 2008), mientras que en otras zonas del Mediterráneo, como Argelia o el norte de Italia, no se conoce más que el vuelo de primavera (Chakali 2005; Faccoli et al., 2005).

Estos picos de vuelo se corresponden con sendos vuelos reproductivos que van a ocasionar un máximo de dos generaciones hermanas. De acuerdo con datos no publicados sobre trabajos realizados en Murcia entre 2001 y 2002, las hembras capturadas durante el primer pico vuelo reproductivo, entre noviembre y diciembre, se encontraban en el estado gonadal C. De acuerdo con Lanströng (1983), en este estado las gónadas presentan oocitos en diferente estado de madurez, pero no presentan corpora lutea. Esto quiere decir que estas hembras no habían realizado aún ninguna puesta. En cambio en el segundo pico de vuelo reproductivo, entre diciembre y marzo, la mayor parte de las hembras capturadas presentaban corpora lutea, el estado D descrito por Lanströng (1983), lo que indica que esas hembras habían ya realizado al menos una puesta. Esto confirma que T. destruens tiene capacidad de desarrollar al menos (y posiblemente como mucho) dos generaciones hermanas, cada una tras sendos picos de vuelo reproductivo, con un periodo de remaduración intermedio.

T. destruens es una especie de hábitos monógamos, siendo la hembra la que ini- 
cia la galería reproductora (unirramea vertical), tras lo que acude el macho. En el primer vuelo reproductivo, tras la cópula en la cámara nupcial, la hembra continúa excavando la galería, colocando los huevos a ambos lados de la misma. El macho permanece en la parte posterior de la galería, ayudando a evacuar el serrín producido durante la excavación. Pasado un tiempo el macho abandona la galería y se dirige a las copas donde pasará un tiempo indeterminado hasta remadurar y estar disponible para una segunda cópula. La hembra permanece más tiempo en la galería, incluso acabada la puesta, aunque llegado el momento, también la abandona, dirigiéndose a las copas para alimentarse hasta el siguiente vuelo reproductivo. Esto implica que los adultos permanecerán juntos en la galería únicamente durante un periodo corto de tiempo, no muy bien determinado.

El levante de la península Ibérica ha registrado una sequía extrema desde la primavera de 2013 hasta otoño de 2015, dando lugar a un profundo deterioro del estado fitosanitario de los pinares valencianos. Esta situación ha supuesto un aumento de la disponibilidad de material vegetal hospedante para insectos perforadores, entre ellos T. destruens. El control de estos insectos se basa en dos herramientas de gestión: el uso de puntos cebo y las trampas cebadas con atrayentes cairomonales, etanol y $\alpha$-pineno (Gallego et al., 2004, Kelsey et al., 2014). Tradicionalmente los puntos cebo han sido utilizados como herramienta en la gestión de los focos de esta especie, pero su elaboración es costosa y su manejo complicado, ya que bien requiere que sean tratados con piretroides insecticidas autorizados (aunque la interpretación de si el uso autorizado en pinos lo está también para trozas de pinos está pendiente), bien deben ser destruidos tras la máxima colonización y antes de la emergencia de los juveniles. En los últimos años se han comercializado atrayentes cairomonales (etanol y $\alpha$-pineno) para ser utilizados en trampas como alternativa eficaz a las pilas cebo. El presente trabajo pretende exponer los resultados obtenidos en la comparación de la eficacia en la extracción de $T$. destruens entre puntos cebo y trampas cebadas con atrayentes cairomonales en tres zonas de la provincia de Valencia, desde el otoño de 2014 a la primavera de 2015, con el objetivo de mejorar la gestión de las poblaciones de este insecto en los pinares valencianos.

\section{Material y métodos}

Entre el 14 de noviembre y el 18 de marzo de 2015 se instalaron 63 dispositivos en tres localidades de la Comunidad Valenciana afectadas por mayor o menor grado de mortandad de pinar producida por T. destruens. En total se instalaron 30 puntos cebo y 33 trampas cebadas, que incluían 22 trampas tipo cross-vane (Crosstrap ${ }^{\circledR}$, Econex, Murcia) y 11 trampas tipo multiembudos (varios proveedores). Los cebos utilizados siempre fueron los suministrados por Sanidad Agrícola Econex S.L. (Murcia), compuestos por un dispensador de etanol, con una emisión de $2000 \mathrm{mg} /$ día y un dispensador de $\alpha$-pineno, con una emisión de $300 \mathrm{mg} /$ día, ambos con una duración de 60 días. Los puntos cebo consistieron en el apilamiento de entre 6 y 17 trozas provenientes de uno o varios ejemplares de pino carrasco recién apeados. La lon- 
gitud media de las trozas fue de $89 \mathrm{~cm}$ y el diámetro medio de $11 \mathrm{~cm}$. Detalles de la instalación se ilustran en la Tab. 1. En la localidad de Villamarxant se instalaron 28 dispositivos, en la de Jalance 10 y en la de Calles 25.

Las trozas se instalaron el 14 y 19 de noviembre de 2014 y fueron retiradas el 6, 12 y 18 de marzo y el 22 de abril de 2015. El examen de las trozas se realizó a la retirada de éstas, mediante el descortezado y recuento exhaustivo de las galerías. Con ello se pretendía conocer el número máximo de galerías (esto es, de parejas) que podrían ocupar las trozas de modo que si se destruyeran en el momento de máxima ocupación, se extraería de la población el mayor número de adultos de $T$. destruens. Se asumió que el número máximo de adultos que ocuparon los cebos en un momento dado fue el doble del número de galerías (una pareja por galería). Esta estima se define como Número Máximo de Adultos (NMA) y va a ser utilizado en este trabajo como número comparativo al número de insectos capturados en trampas.

Las trampas se revisaron semanalmente entre el 14 de noviembre de 2014 y el 22 de abril de 2015, vaciando las capturas en un vial que contenía etanol al $70 \%$, hasta su procesado en laboratorio, donde se realizó el recuento directo de los individuos de T. destruens capturados.

Para las comparaciones entre trampas y cebos se utilizaron únicamente los registros de capturas en las fechas coincidentes entre la instalación y retirada de los cebos en cada localidad (Tabla 1).

Tabla 1. Número de trampas y cebos instalados por localidad.

\begin{tabular}{lcccc}
\hline & Localidad 1 & Localidad 2 & Localidad 3 & \\
\hline & Villamarxant & Jalance & Calles & Total \\
\hline & $\begin{array}{c}\text { De } 14 / 11 / 2014 \\
\text { a } 6 / 3 / 2015\end{array}$ & $\begin{array}{c}\text { De } 19 / 11 / 2014 \\
\text { a } 18 / 3 / 2015\end{array}$ & $\begin{array}{c}\text { De } 19 / 11 / 2014 \\
\text { a } 18 / 03 / 2015\end{array}$ & \\
\hline Puntos cebo & 13 & 6 & 11 & 30 \\
Crosstrap $^{\circledR}$ & 7 & 4 & 11 & 22 \\
$\begin{array}{l}\text { Trampas } \\
\text { multiembudo }\end{array}$ & 8 & 3 & 0 & 11 \\
\hline Total & $\mathbf{2 8}$ & $\mathbf{1 3}$ & $\mathbf{2 2}$ & $\mathbf{6 3}$ \\
\hline
\end{tabular}

\section{Procedimiento analítico}

Los resultados fueron analizados mediante ANOVA-GLM, utilizando diferentes extensiones de R (R Development Core Team, 2015). En el caso de que se detectaran diferencias significativas, se aplicaría un test de Tukey con ajuste de Bonferroni. El número de repeticiones de cada tratamiento (cebos, trampas cross-vane, trampas multiembudos) no estaba balanceado (Tab. 1), por lo que para permitir el ANOVA-GLM se diseñó un procedimiento de muestreo sin reemplazamiento similar al de Montecarlo. Este procedimiento de muestreo consistió en la extracción una 
muestra aleatoria de tamaño 5 de cada tratamiento, sobre la que se calculó la media. Estos valores se archivaron en una matriz de datos. El procedimiento se repitió 100 veces con lo que la matriz final tenía un tamaño de 100 filas y 3 columnas (una por tratamiento). Sobre esta matriz se realizó el ANOVA-GLM, una vez comprobada la normalidad y homocedasticidad de la distribución de los datos.

\section{Resultados}

El número total de ejemplares de T. destruens capturados en trampas han sido de 3654 , a razón de 2333 en trampas Crosstrap ${ }^{\circledR}$ y 1321 en trampas multiembudo (Tab. 2). En el total de trozas se han contabilizado 1443 galerías, que se corresponde con el número de parejas que ocuparon en algún momento los cebos. A partir de este número puede estimarse que el NMA de insectos reproductores que han podido estar bajo la corteza de los cebos en el momento de máxima ocupación fue de 2886 ejemplares.

Tabla 2. Número total de ejemplares de T. destruens capturados en trampas y estimados en cebos. Entre paréntesis se incluyen los resultados de capturas media acumulada ( \pm eem) de insectos atraídos.

\begin{tabular}{|c|c|c|c|c|}
\hline & Villamarxant & Jalance & Calles & Total \\
\hline Puntos cebo & $\begin{array}{c}2348 \\
(181.85 \pm 50.44)\end{array}$ & $\begin{array}{c}412 \\
(50.1 \pm 20.45)\end{array}$ & $\begin{array}{c}126 \\
(9.3 \pm 2.8)\end{array}$ & 2886 \\
\hline Crosstrap $^{\circledR}$ & $\begin{array}{c}1011 \\
(77.14 \pm 29.15)\end{array}$ & $\begin{array}{c}133 \\
(23.01 \pm 11.51)\end{array}$ & $\begin{array}{c}1037 \\
(70.62 \pm 21.3)\end{array}$ & 2333 \\
\hline $\begin{array}{l}\text { Trampas } \\
\text { multiembudo }\end{array}$ & $\begin{array}{c}1163 \\
(82.83 \pm 29.29)\end{array}$ & $\begin{array}{c}310 \\
(107.23 \pm 61.9)\end{array}$ & - & 1321 \\
\hline Total Trampas & 2174 & 443 & 1036 & 3654 \\
\hline Total & 4522 & 855 & 1163 & 6540 \\
\hline
\end{tabular}

${ }^{\text {a }}$ NMA estimado a partir del número de galerías

Cuando se comparan los resultados de capturas entre trampas y cebos, el ANOVA-GLM balanceado detectó diferencias significativas entre el número de insectos capturados en trampas ( 19, $\mathrm{P}_{\text {valor }}=0.000021$ ). Al aplicar el test de Tukey con ajuste de Bonferroni se confirma que el número de insectos capturados en trampas es significativamente mayor que el NMA estimado en cebos. (Fig. 1).

Si se comparan los cebos con los dos tipos de trampa por separado, se detectan diferencias significativas, de forma que ambos tipos de trampas capturan significativamente más ejemplares de $T$. destruens que el NMA estimado para los cebos $(\mathrm{F}=$ 13.3, $\mathrm{P}_{\text {valor }}=0.00000275$ ), como se ilustra en la Fig.2. No se han encontrado diferen- 


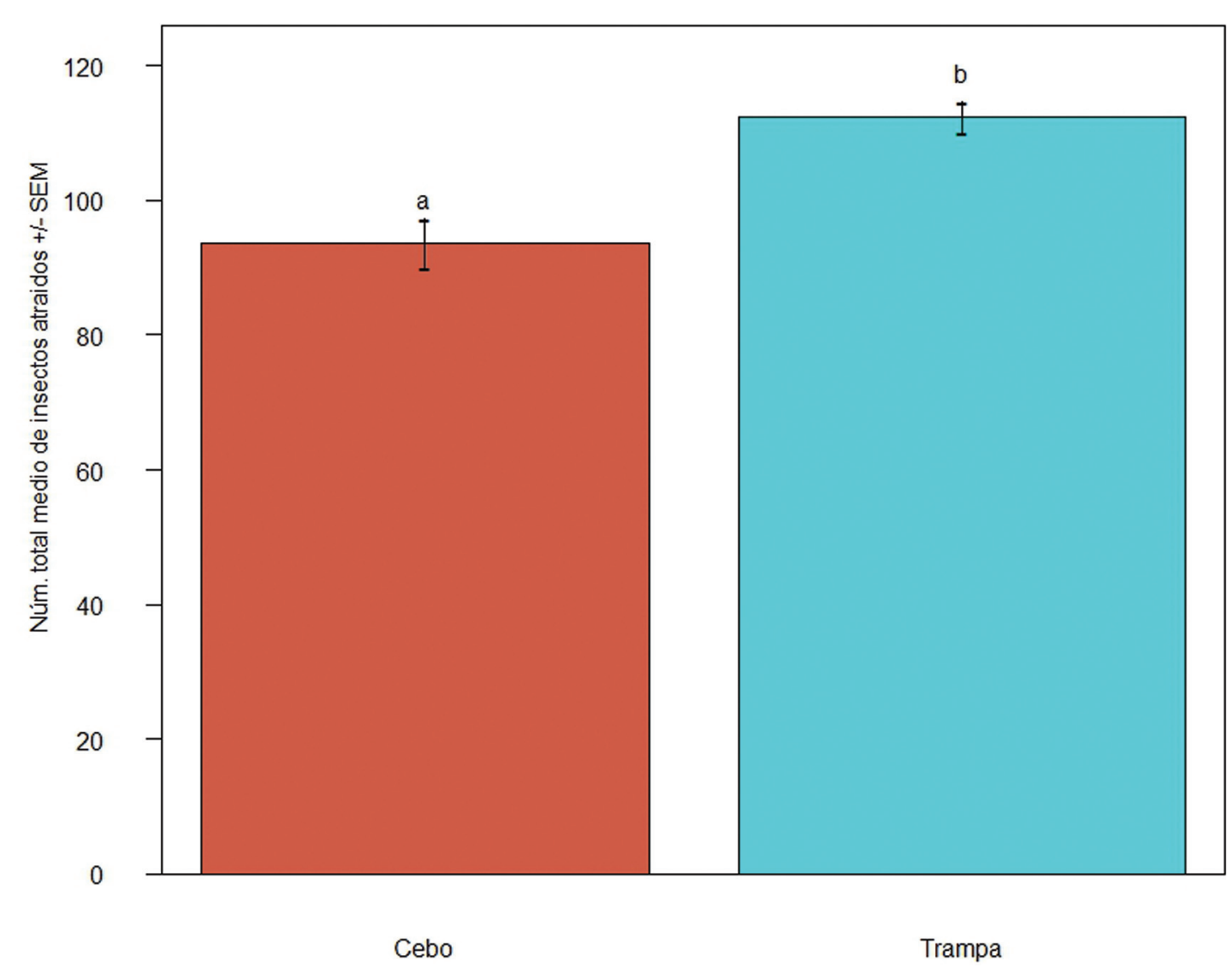

Figura 1. Captura media acumulada ( \pm eem) de insectos atraídos en trampas (ambos tipos) y puntos cebos (NMA estimado a partir del número de galerías). Letras diferentes indican diferencias estadísticamente significativas en ANOVA-GLM, complementado con test de Tukey con ajuste de Bonferroni.

cias significativas en los niveles medios de captura entre ambos modelos de trampa. Las ligeras desviaciones de los resultados medios entre ambas figuras son debidas a los submuestreos del procedimiento empleado para balancear la muestra.

No se ha podido realizar ANOVA-GLM cuando se ha comparado entre NMA de cebos y la atracción media en tipos de trampa por localidad debido al enorme desbalanceo entre tratamientos. Al haberse instalado únicamente 3 trampas multiembudos en la Localidad de Jalance y ninguna en la de Calles, era imposible realizar el submuestreo aleatorio. Entonces únicamente se comparan cualitativamente los resultados de insectos atraídos, ilustrados en la Tab. 2. Se observa una gran variabilidad en resultados. En Calles los cebos apenas han atraído T. destruens, frente a las elevadas capturas en Crosstrap ${ }^{\circledast}$. En cambio, en Jalance, las menores capturas medias se han obtenido en Crosstrap ${ }^{\circledR}$, destacando aquí las altas capturas en trampas multiembudos. En Vilamarxant los NMA en cebos han sido más altos que alguno de los dos modelos de trampa. 


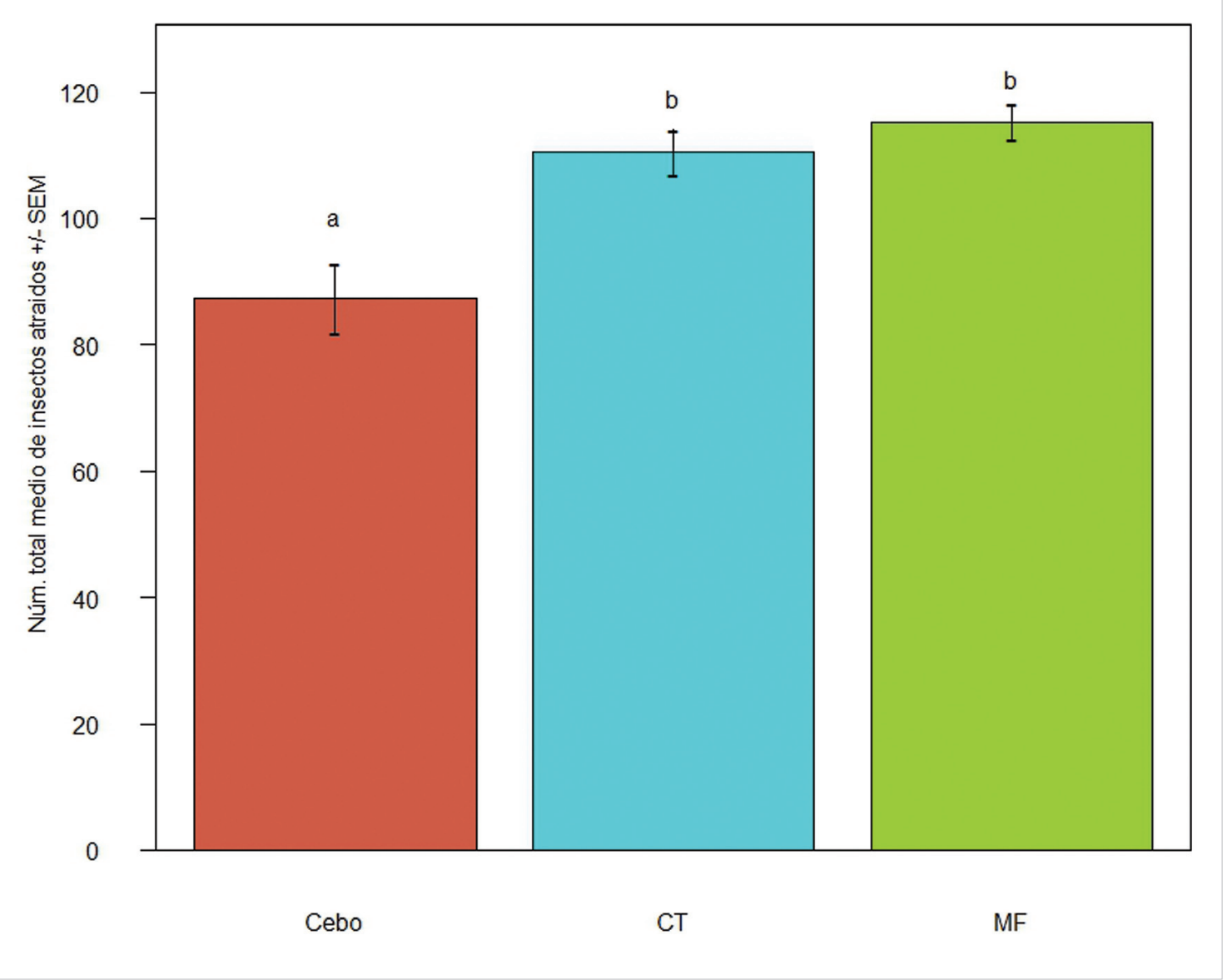

Figura 2. Captura media acumulada ( \pm eem) de insectos atraídos según el tipo trampas y puntos cebos (NMA estimado a partir del número de galerías). Letras diferentes indican diferencias estadísticamente significativas en ANOVA-GLM, complementado con test de Tukey con ajuste de Bonferroni. CT $=$ Crosstrap ${ }^{\circledR}$, $\mathrm{MF}=$ Multiembudos.

\section{Discusión}

Nuestros resultados indican que las capturas son más altas en trampas que la estima de máxima ocupación (NMA) de T. destruens en cebos, independientemente del tipo de trampa. También se detecta una enorme variabilidad en los resultados en los diferentes dispositivos entre localidades. Esta variabilidad no queda explicada por los diferentes niveles poblacionales entre localidades (un $82 \%$ menos de capturas entre Villamarxant y Jalance), ya que no se mantiene el patrón de atracción relativa entre los diferentes dispositivos. De este modo, en Villamarxant el cebo presenta un NMA superior a las capturas medias en las trampas, en las cuales, la captura en Crosstrap ${ }^{\circledR}$ es sensiblemente superior a la obtenida con multiembudos. En cambio en Jalance, con unos niveles de captura muy inferiores, los menores niveles se obtienen en la trampa Crosstrap ${ }^{\circledR}$, siendo los intermedios los obtenidos en el cebo y los superiores (aunque muy variables) en la trampa multiembudos. Por el contrario, en la localidad de Calles, donde no se instalaron trampas multiembudos, es en la trampa Cross- 
$\operatorname{trap}^{\circledR}$ donde se registra un nivel de capturas mucho más elevado que el NMA de los cebos.

Pese a esta variabilidad, se observa una clara tendencia a una mayor eficiencia de las trampas cebadas con etanol y $\alpha$-pineno en la captura de $T$. destruens frente a los cebos. Los dos compuestos semioquímicos atrayentes usados en el cebo se corresponden con la emisión de un pino en fases iniciales de debilitamiento por sequía (Kelsey et al., 2013). De acuerdo con estos autores, el etanol es producido activación del catabolismo anaeróbico en raíces y tejidos profundos del árbol cuando se produce una desconexión entre la copa y las raíces, como es el caso de las sequias extremas, o el tejido vegetal aislado de una troza. Este etanol, un metabolito secundario, no es un mecanismo de defensa de los árboles, pero tiene función cairomonal en la atracción de numerosos escolítidos (Joseph et al., 2001; Kelsey and Joseph, 2001, 2003; Coyle et al., 2005; Gallego et al., 2008; Miller and Rabaglia 2009; Ranger et al., 2011; Kelsey et al., 2013). Además, la liberación de etanol en combinación con otros terpenoides (como $\alpha$-pineno) puede sinergizar la atracción de estas especies (Schroeder and Lindelöw, 1989; Gallego et al.., 2008; Miller and Rabaglia, 2009; Lingren et al., 2012). Por lo tanto, y puesto que se trata de biosíntesis en tejidos vegetales, etanol y $\alpha$-pineno serán emitidos por las trozas del cebo durante el tiempo comprendido entre la tala y la muerte del tejido vegetal. Esto implica que la trampa cebada compita en atracción con el cebo y ambos con los árboles debilitados de forma natural. Y aun así las trampas son más eficientes en la captura que los cebos. Esta mayor eficiencia de la trampa puede deberse a la mayor estabilidad en la emisión de compuestos semioquimicos en la trampa que el cebo. De cualquier modo no puede olvidarse que esta eficiencia relativa se está estimando al comparar el recuento de las capturas de ejemplares reproductores de T. destruens en una trampa, con el número de galerías maternas excavadas con éxito en las trozas de un cebo y multiplicando el número de galerías por dos adultos, lo que se correspondería el momento exacto de NMA.

Hay que resaltar las importantes diferencias en el manejo de trampas y cebos. El uso de las trampas cebadas implica la captura y muerte de adultos reproductores de forma sostenida durante todo el periodo de vuelo del insecto. Los insectos de ambos sexos atrapados son extraídos de la población reproductora y no causan daños en los árboles. En cambio, con el uso de cebos, aunque también se atrae al mismo tipo de adultos reproductores, la atracción no causa la muerte de estos insectos, sino que se ofrece un sustrato vegetal idóneo para la reproducción, siempre y cuando los cebos no sean tratados con insecticidas autorizados, aunque esto no objeto de este trabajo. La gestión del cebo requiere por lo tanto un seguimiento preciso de la curva de llegada de adultos reproductores al mismo para determinar el momento de máxima ocupación, lo que se ha definido en el presente trabajo como NMA, y destruir el cebo y a los adultos que contiene. El problema reside que el intervalo de tiempo para alcanzar el NMA no es constante y depende de diversos factores, entre los que se encuentran la superficie de floema-cambium disponible, el estado de vigor del árbol previo a la corta, el tiempo que tarde en deshidratarse por la exposición a vientos e incidencia del sol, y el grosor de la troza, entre otros. Por lo tanto, la determinación 
del momento NMA resulta muy difícil sin levantar la corteza de la troza, lo que implica la destrucción parcial de la misma. Si la troza se destruye antes del momento NMA se pierde potencial de atracción. En cambio, si se destruye después del momento NMA, una parte de los adultos reproductores habrá abandonado la troza. Cuanto más se aleje la destrucción de la troza del momento NMA, mayor será el número de insectos que abandonen la misma, comenzando también la salida de los juveniles, momento a partir del cual el cebo pierde la función de control poblacional.

\section{Conclusiones}

En conclusión, el uso de trampas cebadas para la retirada de población de T. destruens representa ventajas sobre el uso de puntos cebo:

- Mayor eficiencia media de capturas.

- Retirada efectiva y sostenida de la población de los individuos atrapados, sin posibilidad de retorno.

- Mayor sencillez de instalación.

- Gran facilidad de mantenimiento.

\section{Bibliografía}

Coyle, D.R., Booth, D.C., Wallace, M.S. ,2005. Ambrosia beetle (Coleoptera: Scolytidae) species, flight, and attack on living eastern cottonwood trees. J. Econ. Entomol. 98, 2049-2057. doi:10.1603/0022-0493-98.6.2049.

Chakali G., 2005. L'Hylésine des Pins, Tomicus destruens (Wollaston, 1865) (ColeopteraScolytidae) en Zone Semi-Aride (Algérie). Silva Lusitana. 13: 113-124.

Faccoli M., Battisti A., Masutti L., 2005. Phenology of Tomicus destruens (Wollaston) in northern Italian pine stands. In: Lieutier F., Ghaioule D. Entomological research in Mediterranean Forest Ecosystems, pp .185-193. Institut National de la Recherche Agronomique.

Fäldt J., 2000. Volatile constituents in conifers and conifer related wood-decaying fungi. $\mathrm{PhD}$ These. Royal Institute of Technology, Stockholm.

Filella I., Peñuelas J., Seco R., 2009. Short-chained oxygenated VOC emissions in Pinus halepensis in response to changes in water availability. Acta Physiologiae Plantarum 31, 311-318. doi: 10.1007/s11738-008-0235-6.

Gallego D., 2006. Tomicus destruens (Wollaston, 1865) (Coleoptera, Scolytidae), un estudio a múltiple escala. Tesis Doctoral. Universidad de Murcia, Murcia.

Gallego D., Canovas F., Esteve M., Galian J., 2004. Descriptive biogeography of Tomicus (Coleoptera: Scolytidae) species in Spain. J. Biogeog. 31, 2011-2024. doi: 10.1111/j. 1365-2699.2004.01131.x

Gallego D., Galián J., 2008. Hierarchical structure of mitochondrial lineages of Tomicus destruens (Coleoptera, Scolytidae) related to environmental variables. J. Zool. Syst. Evol. Res. 46, 331-339. doi: 10.1111/j.1439-0469.2008.00472.x 
Gallego D., Galián J., Diez J.J., Pajares J.A., 2008. Kairomonal responses of Tomicus destruens (Col., Scolytidae) to host volatiles a-pinene and ethanol. J. App. Ent. 132, 654-662. doi: 10.1111/j.1439-0418.2008.01304.x

Joseph, G., Kelsey, R.G., Peck, R.W., Niwa, C.G., 2001. Response of some scolytids and their predators to ethanol and 4-allylanisole in pine forests of central Oregon. J. Chem. Ecol. 27, 697-715. doi: 10.1023/A:1010345817756.

Kelsey, R.G., and Joseph, G., 2001. Attraction of Scolytus unispinosus bark beetles to ethanol in water-stressed Douglas-fir branches. For. Ecol. Manage. 144, 229-238. doi:10.1016/ S0378-1127(00)00387-X.

Kelsey, R.G., and Joseph, G., 2003. Ethanol in ponderosa pine as an indicator of physiological injury from fire and its relationship to secondary beetles. Can. J. For. Res. 33, 870 -884. doi:10.1139/x03-007.

Kelsey, R.G., Gallego, D., Sánchez-García F.J., Pajares, J.A., 2014. Ethanol accumulation during severe drought may signal tree vulnerability to detection and attack by bark beetles. Can. J. For. Res., 44: 554-561. doi: 10.1139/cjfr-2013-0428

Langström, B., 1983 Life cycles and shoot-feeding of the pine shoot beetles. Studia Forestalia Suecica, 163, 1-29.

Lingren, B.S., Miller, D.R., and LaFontaine, J.P., 2012. MCOL, frontalin and ethanol: a potential operational trap lure for Douglas-fir beetle in British Columbia. J. Entomol. Soc. B.C. 109, 71-73.

Miller, D.R., Rabaglia, R.J. 2009. Ethanol and (-)- $\alpha$-pinene: attractant kairomones for bark and ambrosia beetles in the southeastern US. J. Chem. Ecol. 35, 435-448. doi:10.1007/ s10886-009-9613-9.

Sabbatini Peverieri G., Faggi M., Marziali L., Tiberi R., 2008. Life cycle of Tomicus destruens in a pine forest of central Italy. Bull. Insectology. 61, 337-342.

Schroeder, L.M., and Lindelöw, Å,. 1989. Attraction of scolytids and associated beetles by different absolute amounts and proportions of $\alpha$-pinene and ethanol. J. Chem. Ecol. 15, 807-817. doi:10.1007/BF01015179.

R Development Core Team, 2015. R: A language and environment for statistical computing. R Foundation for Statistical Computing. ISBN: 3-900051-07-0. Available online at http: //www.R-project.org/.

Ranger, C.M., Reding, M.E., Gandhi, K.J.K., Oliver, J.B., Schulty, P.B., Cañas, L., Herms, D.A. 2011. Species dependent influence of (-)- $\alpha$-pinene on attraction of ambrosia beetles (Coleoptera: Curculionidae: Scolytinae) to ethanol baited traps in nursery agroecosystems. J. Econ. Entomol. 104, 574-579. doi: 10.1603/EC10243.

Vasconcelos T., Horn A., Lieutier F., Branco M., Kerdelhué C., 2006. Distribution and population genetic structure of the Mediterranean pine shoot beetle Tomicus destruens in the Iberian Peninsula and Southern France. Agr. For. Ent. 8: 103-111. doi: 10.1111/j.14619563.2006.00292.x

Wood, S.L., Bright, D.E., 1992. A catalog of Scolytidae and Platypodidae (Coleoptera). Part 2. Taxonomic index. Great Basin naturalist memoirs, 13, 835-1557. 